\title{
Modified Temperature-Anisotropy Instability Thresholds in the Solar Wind
}

\author{
R. Schlickeiser, ${ }^{1,2}$ M. J. Michno, ${ }^{1}$ D. Ibscher, ${ }^{1}$ M. Lazar, ${ }^{1}$ and T. Skoda ${ }^{1}$ \\ ${ }^{1}$ Institut für Theoretische Physik, Lehrstuhl IV: Weltraum- und Astrophysik, Ruhr-Universität Bochum, \\ D-44780 Bochum, Germany \\ ${ }^{2}$ Research Department Plasmas with Complex Interactions, Ruhr-Universität Bochum, D-44780 Bochum, Germany
}

(Received 26 July 2011; published 11 November 2011)

\begin{abstract}
The proton and electron temperature anisotropies in the solar wind are constrained by the instability thresholds for temperature-anisotropy-driven kinetic plasma instabilities. The modifications to the marginal instability conditions from accounting for the influence of damping connected with the collisional effects in the solar wind plasma are calculated for right- and left-handed polarized parallel propagating Alfvén waves and mirror and firehose fluctuations. These modifications provide tighter threshold constraints compared to the marginal thresholds but do not fully explain the observations at small values of the parallel plasma beta.
\end{abstract}

DOI: 10.1103/PhysRevLett.107.201102

PACS numbers: 95.30.Qd, 52.35.Ra, 96.50.Ci

The explanation of the relaxation of cosmic collisionpoor plasmas with velocity-anisotropic distribution functions (VADs) and their near energy density equipartition with electromagnetic plasma turbulence are two challenging fundamental problems of plasma astrophysics. Thermalization by elastic two-body Coulomb collisions is orders of magnitudes too slow as compared to interactions with electric and magnetic fields because of the low density of cosmic plasmas. The plasma parameter $g=$ $\nu_{e e} / \omega_{p, e}$ is the ratio of the electron-electron Coulomb collision frequency $\nu_{e e}$ to the electron plasma frequency $\omega_{p, e}$, which characterizes interactions with the electromagnetic turbulence. In all cosmic plasmas, including the solar wind, the interstellar and intergalactic medium, and clusters of galaxies, the plasma parameter is smaller than $10^{-10}$, indicating that elastic collisions are not effective in establishing a local thermodynamic equilibrium. These cosmic plasmas can be regarded as effectively collisionpoor on the shortest time and length scales. Alternative relaxation mechanisms, different from thermalization by elastic two-body Coulomb collisions, have to be examined such as the momentum diffusion by second-order Fermi interactions of charged particles with electromagnetic turbulence which is an intrinsic property of any sufficiently agitated magnetized plasma [1,2] with VADs.

The occurrence of VADs is a well-established feature of the solar wind plasma from both the observational [3-5] and theoretical points of view [6]. In theory, this has been attributed to the Chew-Goldberger-Low mechanism [7]: As the wind expands, the plasma density and magnetic field decrease radially. If the particle motion is adiabatic and collisionless, the plasma particles become anisotropic in the sense that the pressure along the magnetic field $P_{\|} \neq P_{\perp}$ differs from the perpendicular pressure. The resulting pressure-anisotropy instabilities [8] then counterbalance by pitch-angle scattering the Chew-GoldbergerLow mechanism, so that nearly isotropic plasma distribution results. Observationally, the solar wind plasma is the only cosmic plasma where detailed in situ satellite observations of plasma properties are available [4]. Although the detailed plasma relaxation processes are not understood, the observed electron and proton distribution functions are close to bi-Maxwellian velocity distributions with different temperatures along and perpendicular to the ordered magnetic field direction. Bi-Maxwellians are special cases of VADs.

Ten years of Wind spacecraft solar wind experiment data [3] near 1 A.U. have demonstrated that the proton and electron temperature anisotropies $A=T_{\perp} / T_{\|}$are bounded by the mirror and firehose instabilities [9] at large values of the parallel plasma beta $\beta_{\|}=8 \pi n k_{B} T_{\|} / B^{2} \geq 1$ (see Fig. 2 below). With marginal linear instability conditions $(\gamma>0)$, it has been demonstrated [10] that the confinement limits at small values of the parallel plasma beta $\beta_{\|}<1$ in principle can be provided by polarized parallel propagating Alfvén waves generated by the bi-Maxwellian proton and electron distributions, although the confinement limits for marginal instability $\gamma>0$ did not match the Wind spacecraft solar wind experiment observations particularly well.

It is the purpose of this work to improve on the agreement of the Alfvénic confinement limits with the solar wind observations near 1 A.U. by properly accounting for the influence of damping processes connected with the collisional effects in the solar wind plasma. Although the plasma parameter of the fully ionized solar wind plasma is small, collisional damping associated with Joule dissipation as well as electron and ion viscosity $[11,12]$ cannot be neglected especially at large wave number values [13]. In contrast to earlier work [10], where the marginal instability condition $\gamma_{R, L}>0$ of parallel propagating right $(R)$ - and left $(L)$-handed polarized Alfvén waves has been used, we consider here the more appropriate instability condition that 


$$
\gamma_{R, L}>\gamma_{\text {damp }}
$$

has to be greater than all collisional damping rates $\gamma_{\text {damp }}$.

At large wave number $(k)$ values, the collisional damping rate is dominated by viscous damping [Eq. (27) of Ref. [13]]

$$
\gamma_{\mathrm{damp}}=3.2 \times 10^{-15} \frac{\beta_{\|} k^{2} c^{2}}{T_{5}^{3 / 2}} \mathrm{~Hz},
$$

for an assumed solar wind proton temperature of $10^{5} T_{5} \mathrm{~K}$.

For the linear growth rate of parallel propagating left(LH) and right-handed (RH) polarized Alfvén waves in a bi-Maxwellian proton or electron plasma of equal parallel temperature and equal electron-proton temperature anisotropy $A$, we use the existing analytical expression $[10,14]$

$$
\gamma_{R, L}=0.477 \frac{h^{6} B_{4}(\mu x)^{3}}{\beta_{\|}\left[1 \pm \frac{1}{2} x \mu\right]} M_{R, L} e^{-\left(h^{2} / \beta_{\|}\right)(1 \pm \mu x)^{2}},
$$

with

$$
M_{R, L}= \pm(1-A)-A x \mu .
$$

$M_{R, L}>0$ characterizes the marginal instability condition. $\mu=m_{p} / m_{e}=1836$ denotes the mass ratio, $\beta_{\|}$the parallel plasma beta, and $x=\omega_{R} /\left|\Omega_{0, e}\right|$ the dimensionless real part of Alfvén wave frequency. The solar wind magnetic fluctuations reported [4] near 1 A.U. have wave numbers $k \simeq \alpha / \rho_{p}$ with $\alpha=0.56 \pm 0.32$ and the thermal proton gyroradius $\rho_{p}=4.23 \times 10^{6} T_{5}^{1 / 2} B_{4}^{-1} \mathrm{~cm}$, where we adopt an interplanetary magnetic field value $B=10^{-4} B_{4} \mathrm{G}$, and therefore correspond to values of the proton plasma frequency phase speed $h=\omega_{p, e} /\left(\mu^{1 / 2} k c\right) \in[2.1,7.7]$. With the observed wave number, we obtain for the damping rate (2)

$$
\gamma_{\mathrm{damp}}=5.1 \times 10^{-8} \frac{\beta_{\|} B_{4}^{2}}{T_{5}^{5 / 2}} \mathrm{~Hz} .
$$

By using Eqs. (3) and (5), the modified instability condition (1) becomes

$$
M_{R, L}>\frac{B_{4}\left[1 \pm \frac{1}{2} x \mu\right]}{T_{5}^{5 / 2} h^{6}(\mu x)^{3}} F\left(\beta_{\|}\right)
$$

with

$$
F\left(\beta_{\|}\right)=\beta_{\|}^{2} e^{-K_{0}+\left(h^{2} / \beta_{\|}\right)(1 \pm \mu x)^{2}}
$$

and $K_{0}=16.1$.

The Alfvénic dispersion relation for $h \gg 0.5$

$$
\mu x_{R, L} \simeq \frac{\sqrt{1+(A-1) \beta_{\|}}}{h}
$$

is identical for left- and right-handed polarized Alfvén waves but requires for values of $A<1$ the existence condition

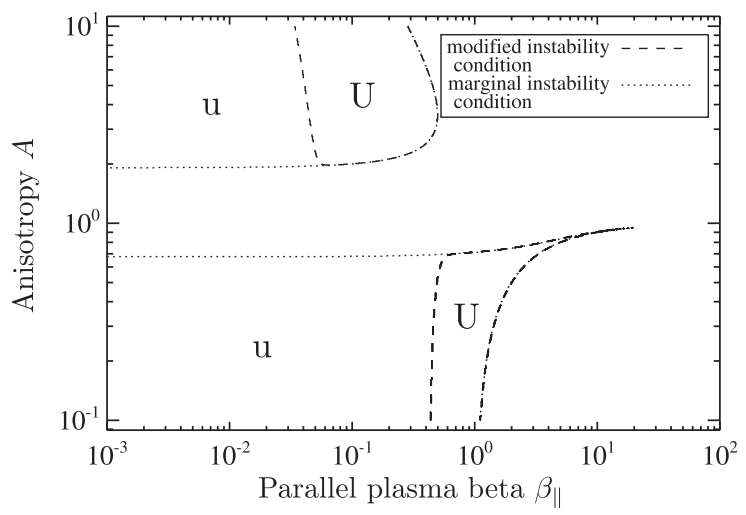

FIG. 1. Anisotropy diagram for parallel propagating LH and $\mathrm{RH}$ polarized Alfvén waves and firehose fluctuations for the case of equal electron and proton temperature anisotropies resulting from the marginal (dotted lines) and modified (dashed lines) instability conditions, calculated for $h=2.1$. Unstable regions from the modified and marginal instability conditions are marked by "U" and "u," respectively.

$$
A>1-\beta_{\|}^{-1},
$$

which coincides with the instability condition of firehose fluctuations [15].

In Fig. 1, we show the anisotropy diagram for $\mathrm{LH}$ and RH polarized Alfvén waves resulting from the marginal instability condition $M_{R, L}>0$ in comparison with the modified instability condition (6), calculated for the value $h=2.1$. The firehose instability condition (9) is also indicated. We notice that the modified instability condition provides much tighter restrictions than the marginal instability condition on the values of $A$ and $\beta_{\|}$for which LH and RH Alfvén waves are unstable.

In Figs. 2 and 3, we compare the anisotropy diagram for LH and RH polarized Alfvén waves, resulting from the modified instability condition (6), calculated for the values $h=2.1$ and $h=0.5$, respectively, in comparison with the

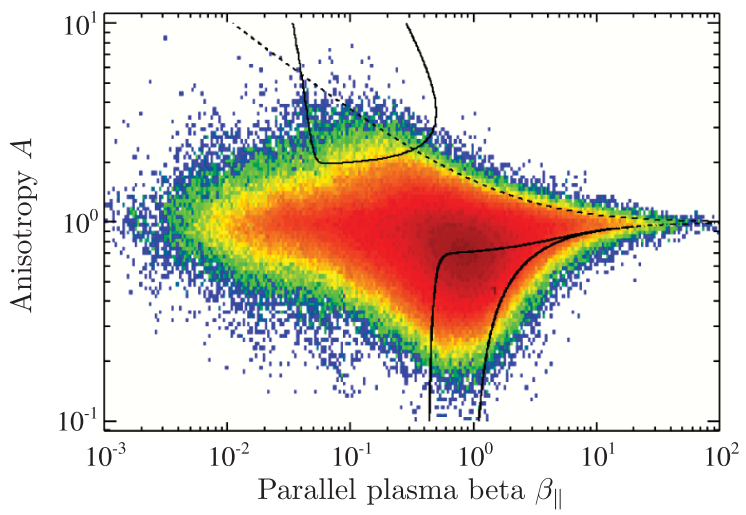

FIG. 2 (color). Modified threshold conditions calculated for $h=2.1$ for parallel propagating $\mathrm{LH}$ and $\mathrm{RH}$ polarized Alfvén waves, firehose (full lines), and mirror (dashed line) fluctuations in comparison with solar wind observations [4]. 


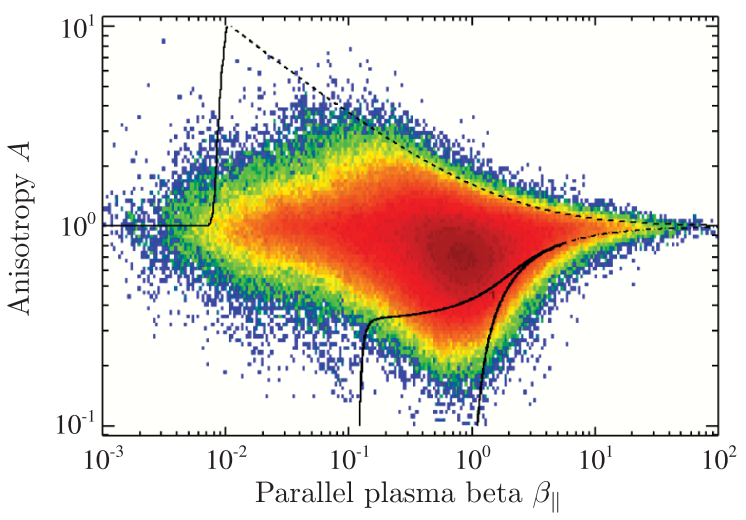

FIG. 3 (color). The same as Fig. 2 for $h=0.5$.

solar wind observations [4]. For large values of the parallel plasma beta, we use the marginal instability conditions of the firehose and the mirror fluctuations [15], assuming that these weakly propagating fluctuations are not affected by the collisional viscous damping processes [16]. We note that for the smaller value of $h$ the instability regions extend to smaller values of $\beta_{\|}$in agreement with Eq. (12) below.

Compared to the earlier Alfvénic marginal instability conditions [10], the modified instability condition (6), accounting for the collisional damping of Alfvén waves, provides a better explanation of the observations at small values of the parallel plasma beta $\beta_{\|}<1$ and values of $A>1$. At large temperature anisotropies $A>1$, the combined action of the thresholds for LH polarized Alfvén waves and mirror fluctuations reasonably accounts for the observations at all plasma beta values. However, for small values of $A<1$ and $\beta_{\|}<1$, the modified threshold condition of parallel RH polarized Alfvén waves disagrees with the observations. Optimizing the fitted values of the parameters $h$ and $K_{0}$ might lead to a better agreement.

Although the modified instability condition (6) is rather involved, the numerical results obtained in Figs. 1-3 can be readily analytically reproduced in the case of $\mathrm{RH}$ polarized Alfvén waves approximating the Alfvénic dispersion relation (8) for $A<1$ by $\mu x \simeq 1 / h$. Condition (6) then simplifies for nominal interplanetary parameter values to

$$
M_{R}>\frac{1+2 h}{h^{4}} \beta_{\|}^{2} \exp \left(-K_{0}+\frac{(h+1)^{2}}{\beta_{\|}}\right),
$$

implying with the existence condition (9)

$$
\begin{gathered}
1-\frac{1}{\beta_{\|}}<A<Y\left(h, \beta_{\|}\right), \\
Y\left(h, \beta_{\|}\right)=\frac{h}{h+1}\left[1-\frac{1+2 h}{h^{4}} \beta_{\|}^{2} e^{-K_{0}+\left[(h+1)^{2} / \beta_{\|}\right]}\right] .
\end{gathered}
$$

The function $Y\left(h, \beta_{\|}\right)$is tighter than the marginal instability condition $Y=h /(h+1)$. The function $Y\left(h, \beta_{\|}\right)$is negative for values $\beta_{\|}<\beta_{0}(h)$ with

$$
\beta_{0}(h) \simeq \frac{(h+1)^{2}}{K_{0}+\ln \frac{h^{4}}{1+2 h}}
$$

which increases with increasing values of $h$, reaching unity for $h=3.35$. Consequently, for values of $h>3.35 \mathrm{RH}$ polarized Alfvén waves are stable according to the modified instability condition. For values of $0.5 \leq h \leq 3.35$, RH polarized Alfvén waves are unstable for parallel plasma betas greater $\beta_{\|}>0.17$, in agreement with Figs. 1-3 [note that $\left.\beta_{0}(2.1)=0.55\right]$. For values $\beta_{\|}>\beta_{0}$ the function $Y\left(h, \beta_{\|}\right)$steeply increases up to the value $h /(h+1)$.

Our calculations therefore suggest the following physical explanation: In the parameter plane defined by the temperature anisotropy $A=T_{\perp} / T_{\|}$and the parallel plasma beta $\beta_{\|}$, stable plasma configurations are possible only within a rhomblike configuration around $\beta_{\|} \simeq 1$, whose limits are defined by the marginal threshold conditions for the mirror and firehose instabilities at large values of $\beta_{\|}$and by the modified threshold condition for the Alfvén wave instabilities. If a plasma would start with parameter values outside this rhomblike configuration, it immediately would generate fluctuations via these instabilities, which quickly relax $[17,18]$ the plasma distribution into the stable regime within the rhomb configuration. Within the rhomb configuration, the observed magnetic fluctuations are generated by spontaneous emission $[19,20]$ of the stable particle distribution functions.

As other dilute cosmic plasmas have similar densities, temperatures, and magnetic fields as the solar wind, the suggested relaxation process, via the generation of Alfvén waves and mirror and firehose fluctuations, to stable plasma configurations around $\beta_{\|} \simeq 1$, likely also operates there. Note that $\beta_{\|} \simeq 1$ corresponds to the equipartition of the magnetic field energy density and the parallel kinetic energy density of plasma particles, which is a wellestablished but so-far unexplained property of many cosmic sources including especially the interstellar medium. For the interstellar medium it has been known for a long time [21-23], even before the discovery of the universal cosmic microwave background radiation, that these interstellar components have comparable energy densities and pressures, each of the order of $10^{-12} \mathrm{erg} \mathrm{cm}^{-3}$, commonly referred to as the global equipartition condition in the interstellar medium. Equipartition conditions for the magnetic field energy density and the kinetic energy density of plasma particles in astrophysical sources are also often invoked for convenience [24-26] in order to analyze cosmic synchrotron intensities. Until today, this truly remarkable equipartition has not been understood nor explained theoretically. The explanation of solar wind equipartition conditions by kinetic plasma effects presented here might remedy this unacceptable situation. In this context [27] we emphasize that the level of the partition between the magnetic and (parallel) kinetic energy density in the solar wind is also crucial to understand the observationally reported 
[28-31] steepening of the magnetic field fluctuation power spectra at high frequencies $f \geq 0.5 \mathrm{~Hz}$, in the so-called dissipation range of turbulence. It has been pointed out $[32,33]$ that the difference of power spectra in the dissipation range from those in the inertial range of turbulence at low frequencies can result if the equipartition condition at the fluid level is broken. The also available magnetic field power spectrum (see Fig. 4 of Ref. [4]) indeed suggests such a steepening at large wave number values $k \rho_{p} \geq 2$, although in the wave number range $k \simeq \alpha / \rho_{p}$ with $\alpha=$ $0.56 \pm 0.32$ of the data shown in Figs. 2 and 3 the power spectrum exhibits the Kolmogorov power law form characteristic for the inertial range.

However, extending the existing instability studies in the solar wind to the different interstellar medium phases is highly nontrivial because, most importantly, there are no in situ plasma data available, and additionally several important effects from the influence of cosmic rays and gas neutrals have to be included. First of all, unlike as in the solar wind case, the cosmic ray gas itself serves as an important source of plasma turbulence at nearly all wave numbers by efficient instabilities driven by pitch-angle anisotropies, streaming instabilities, loss-cone distributions in converging magnetic field lines, or inverted energy distributions, especially near powerful cosmic ray point source injectors. Currently, it is an unsettled question whether gas or cosmic ray driven instability threshold conditions define the boundary limits of the stable interstellar medium configurations for different plasma betas.

Second, the presence of neutral gas and molecules in the denser parts of the interstellar medium provides additional efficient damping processes of the generated turbulent magnetic fields by ion-neutral friction [34] that could stabilize the otherwise unstable gas and cosmic ray distributions.

Finally, the generation of VADs for the ionized interstellar gas particles has to be investigated. In the case of the solar wind, this is attributed to the Chew-Goldberger-Low mechanism. Of course, the same mechanism applies to the interstellar medium near stellar winds or supernova remnants, but in the more quieter phases of the interstellar medium alternative mechanisms have to be identified, such as the mentioned momentum diffusion by second-order Fermi interactions of charged gas and dust particles with electromagnetic turbulence. Additionally, strong spatial gradients of the isotropic part of the cosmic ray density near cosmic ray accelerators provide anisotropic cosmic ray velocity distributions.

This work was partially supported by the Deutsche Forschungsgemeinschaft through Grant No. Schl 201/21-1.

[1] E. N. Parker and D. A. Tidman, Phys. Rev. 111, 1206 (1958).

[2] R. Schlickeiser, Astron. Astrophys. 143, 431 (1985).
[3] J. C. Kasper, A. J. Lazarus, and S. P. Gary, Geophys. Res. Lett. 29, 20 (2002)

[4] S. D. Bale, J.C. Kasper, G. G. Howes, E. Quataert, C. Salem, and D. Sundkvist, Phys. Rev. Lett. 103, 211101 (2009).

[5] B. A. Maruca, J.C. Kasper, and S.D. Bale, preceding Letter, Phys. Rev. Lett. 107, 201101 (2011).

[6] E. Marsch, Living Rev. Solar Phys. 3, 1 (2006).

[7] G. F. Chew, M. L. Goldberger, and F. E. Low, Proc. R. Soc. A 236, 112 (1956).

[8] A. Eviatar and M. Schulz, Planet. Space Sci. 18, 321 (1970).

[9] P. Hellinger, P. Travnicek, J. C. Kasper, and A. J. Lazarus, Geophys. Res. Lett. 33, L09101 (2006).

[10] R. Schlickeiser and T. Skoda, Astrophys. J. 716, 1596 (2010).

[11] S. I. Braginskii, Rev. Plasma Phys. 1, 205 (1965).

[12] J. V. Hollweg, J. Geophys. Res. 90, 7620 (1985).

[13] M. Lazar, F. Spanier, and R. Schlickeiser, Astron. Astrophys. 410, 415 (2003).

[14] S.P. Gary, Theory of Space Plasma Microinstabilities (Cambridge University Press, Cambridge, England, 1993).

[15] R. Schlickeiser, M. Lazar, and T. Skoda, Phys. Plasmas 18, 012103 (2011).

[16] For firehose fluctuations the modified instability conditions $A<1-\beta_{\|}^{-1}-9.7 \times 10^{-5} \beta_{\|}^{1 / 2} h B_{4}^{2} T_{5}^{-5 / 2}$ is only slightly different from the marginal instability condition $A<1-\beta_{\|}^{-1}$.

[17] P. Yoon, Phys. Plasmas 14, 102302 (2007).

[18] L. Matteini, S. Landi, M. Velli, and P. Hellinger, J. Geophys. Res. 115, A09106 (2010).

[19] P. Yoon, Phys. Plasmas 14, 064504 (2007).

[20] R. C. Tautz and R. Schlickeiser, Phys. Plasmas 14, 102102 (2007).

[21] L. Biermann and L. Davies, Z. Astrophys. 51, 19 (1960).

[22] E. N. Parker, Astrophys. J. 145, 811 (1966).

[23] E. N. Parker, in Nebulae and Interstellar Matter, edited by B. M. Middlehurst and L. H. Aller (University of Chicago, Chicago, 1968), p. 707.

[24] G. R. Burbidge, Astrophys. J. 124, 416 (1956).

[25] M.S. Longair, Particles, Photons and Their Detection, High Energy Astrophysics Vol. 1 (Cambridge University Press, Cambridge, 1992).

[26] R. Beck and M. Krause, Astron. Nachr. 326, 414 (2005).

[27] We are grateful to the referee for noting this subject.

[28] C. W. Smith, K. Hamilton, B. J. Vasques, and R. J. Leamon, Astrophys. J. 645, L85 (2006).

[29] K. H. Kiyanoi, S. C. Chapman, Yu. V. Khotyaintsev, M. W. Dunlop, and F. Sahraoui, Phys. Rev. Lett. 103, 075006 (2009).

[30] O. Alexandrova, J. Saur, C. Lacombe, A. Mangeney, J. Mitchell, S. J. Schwartt, and P. Roberts, Phys. Rev. Lett. 103, 165003 (2009).

[31] F. Sahraoui, M.L. Goldstein, P. Roberts, Yu. V. Khotyaintsev, Phys. Rev. Lett. 102, 231102 (2009).

[32] S. Galtier and E. Buchlin, Astrophys. J. 656, 560 (2007).

[33] Y. W. Jiang, S. Liu, and V. Petrosian, Astrophys. J. 698, 163 (2009).

[34] R. M. Kulsrud and W. P. Pearce, Astrophys. J. 156, 445 (1969). 